\title{
Detecting Mastitis of Dairy Cows with Visible Spectrum
}

\author{
Chien-Hsing Chen ${ }^{*}$ and Chia-Heng Liou \\ Department of Information Technology, Meiho University, 23, Pingguang Rd., Neipu, Pingtung 912, Taiwan
}

(Received July 7, 2017; accepted November 2, 2017)

Keywords: mastitis, California Mastitis Test, visible spectrum

In this study, visible spectra are utilized to investigate the gel formation in the California Mastitis Test (CMT) for dairy cows. The milk and CMT solution will gel in proportion to the number of somatic cell count (SCC) in the milk. Most somatic cells are leukocytes (white blood cells), and they can reveal the severity of udder inflammation. Therefore, CMT is a simple cow-side indicator of SCC in milk. The test does, however, have a few limitations, such as including the subjective assessment of the strength of the gelling reaction between the gelling of the milk sample and the reagent, and it requires some time, slowing down parlor throughput. Also, the relationship between the subjective rating and the SCC (degree of udder infection) is not sufficiently accurate. Therefore, in this study, we objectively identify the relationship between the CMT gelling reaction and SCC using the nondestructive optical inspection technique to achieve a rapid and efficient online determination of the degree of udder infection. The investigation of parameters involves the absorption and transmittance of light that passes through the CMT solution with various SCCs. The intensity decreases systematically as the SCC increases, and it effectively predicts the SCC around $517 \mathrm{~nm}$, but poorly predicts lower SCCs $\left(<10 \times 10^{4}\right.$ cells $\left./ \mathrm{ml}\right)$ around $609 \mathrm{~nm}$. This optical system using the visible spectrum may be utilized as an alternatively potential method for evaluating the CMT score.

\section{Introduction}

Mastitis is undoubtedly one of the most serious and costly infectious diseases that affects dairy cow production. ${ }^{(1)}$ Although the exact cost is difficult to quantify, the estimated cost in the USA in 2009 was $\$ 2$ billion. ${ }^{(2)}$ Clearly, the early diagnosis of mastitis can improve its management, potentially reducing these losses. One obvious symptom of mastitis is an increase in somatic cell count (SCC) in milk; somatic cells are epithelial and white blood cells. The cow's immune system produces somatic cells to fight infection-causing bacteria. ${ }^{(3)}$ Therefore, an automatic cow-side measure of SCC levels would provide a useful indication of mastitis. Measurement of the SCC in raw milk is widely accepted as the most useful indirect indicator of mastitis and milk quality. However, the SCC can be easily obtained in the laboratory using flow cytometry. The instruments that are generally used are large and costly, and can only be operated by trained personnel. The return time for these assays is usually days. To obtain a

*Corresponding author: e-mail: jameschen@meiho.edu.tw

http://dx.doi.org/10.18494/SAM.2018.1753 
faster, but less accurate result, some farmers use a simple, inexpensive, cow-side test, called the California Mastitis Test (CMT) ${ }^{(4)}$ which provides a qualitative estimate of the SCC in the milk of an individual cow in quarters.

CMT is an indirect method of detecting infected quarters on a farm; it is based upon the reaction of special detergents with the DNA of somatic cells. The increase in the viscosity of the mixture is proportional to the SCC, and the degree of gel formation can be evaluated by gently rotating a CMT paddle. However, the test involves a subjective evaluation of the degree of gelling between the milk sample and the reagent, which may result in false positives and negatives. Also, the relationship between the subjective rating and the SCC in the milk sample (an indication of the degree of udder infection) is not sufficiently accurate for many farmers.

To accurately manage the health and diet of an individual cow, a considerably higher sampling rate would be required. Measuring the milk composition in every milking would favor the early detection of systemic and local alterations, providing great inputs for strategic and management decisions. ${ }^{(5)}$ Currently, farmers have access to indirect estimates of the SCC, including online measurements of conductivity, but not to automated online sensors that directly measure SCC levels. The mechanization or standardization of CMT has been attempted using rotary viscometers, ${ }^{(6,7)}$ falling ball viscometers, ${ }^{(8)}$ rolling ball viscometers ${ }^{(9)}$ or some measurement devices that are based on the flow of the CMT gel through holes or tubes of various diameters, as in the Wisconsin Mastitis Test ${ }^{(10)}$ or the Brabant Mastitis Reaction. ${ }^{(11,12)}$ Owing to the need for a short time to implement these processes, potentially slowing down parlor throughput, and none of the aforementioned systems are available for online use.

Fast analytical devices or systems are sought as they would allow an online response to changes in media components. However, most currently used online systems have low accuracy. The CMT gel has been described as thixotropic, ${ }^{(9)}$ meaning that it breaks down under shear force but reforms upon the removal of that force. Also, the rheology of the CMT gel was studied using both capillary and rotational viscometry, ${ }^{(13)}$ revealing that the gel is nonNewtonian, but that the initial viscosity increase is not caused by shear dependence, but to the gelation reaction; therefore, the rheology of the CMT gel is complicated not only by it being non-Newtonian but also by the strong dependence of the test conditions, making it difficult to control and quantify. These facts make designing a successful sensor much more difficult.

Many studies have used optical technology to measure the composition of milk and to detect mastitis. Owing to strong absorbance by $\mathrm{O}-\mathrm{H}$ groups in water, two bands at around 1448 and $1932 \mathrm{~nm}$ dominate the spectra that are obtained by the noninvasive techniques of Fourier transform infrared (FT-IR) and near infrared (NIR) absorption. The characteristic absorption bands of fat, lactose, and protein are much weaker than these two water bands. ${ }^{(14)}$ Two measurement modes (reflectance and transmittance) and different wavelength ranges have been utilized in Vis/NIR spectroscopy and the effectiveness of these methods in measuring the composition of milk online has been compared. ${ }^{(15)}$ The obtained results thus show that the Vis/NIR reflectance measurements support the very accurate monitoring of the fat and crude protein content of raw milk $[R(2)>0.95]$, but poorly predicted the lactose content $[R(2)<0.75]$. In contrast, the Vis/NIR transmittance spectra of the milk samples accurately predict fat and crude protein contents $[R(2)>0.90]$ as well as reasonable lactose predictions $[R(2)=0.88]$. 
In addition, both laser-induced breakdown spectroscopy (LIBS) and laser-induced fluorescence (LIF) techniques were used for diagnosis of mastitis. ${ }^{(16)}$ They showed that the intensities of calcium spectral lines in healthy milk were clearly higher than in mastitic milk samples. On the contrary, the intensities of the two sodium D-lines were higher in mastitic milk than in the healthy milk; however, the time duration of a single sample measurement via LIBS or LIF is nearly $10 \mathrm{~min}$. The most important features of optical systems are robustness, non-invasiveness, precision, low cost, rapidity, and easy automation; therefore, the purpose of this study was to objectively evaluate the CMT score for predicting mastitis by means of visible spectrum, which would become an alternative technique for the online monitoring of mastitis based on CMT.

\section{Materials and Methods}

The currently used CMT is based on the determination of an SCC that exceeds $20 \times 10^{4}$ (cells $/ \mathrm{ml}$ ) for non-clinical mastitis. According to the literature, an SCC of greater than $10 \times$ $10^{4}(\mathrm{cell} / \mathrm{s} / \mathrm{ml})$ indicates that the mammary gland is likely to have been infected by bacteria and therefore to exhibit inflammation. Therefore, the CMT is not very effective in the prevention or early diagnosis in cases of early nonclinical mastitis. In this work, we seek to overcome this shortcoming, and a nondestructive optical detection system is therefore constructed to examine the gelation of CMT solution and to determine the absorption and transmittance of light that passes through a CMT solution with different SCCs.

Figure 1 presents the constructed inspection system. The light source was a halogen lamp (21 $\mathrm{V}, 150 \mathrm{~W}$ ). The width of the single slit was $0.4 \mathrm{~mm}$, and the volume of the glass cuvette with two transparent sides was $1 \mathrm{ml}$, providing a $3 \mathrm{~mm}$-long light path and a wavelength band around $340-2500 \mathrm{~nm}$. The grating in front of the charge-coupled device (CCD) has 1000 lines $/ \mathrm{mm}$, and the intensity of the dispersed light was measured using a CCD.

For purposes of the on-line test, the Microsoft Visual C\# programming language was used to develop the graphical user interface (GUI) of the inspection system, as presented in Fig. 2. This

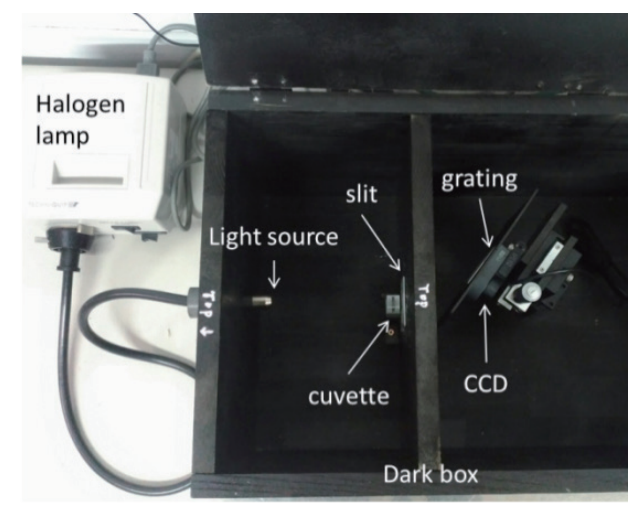

Fig. 1. Experimental setup for visible-spectrum inspection system

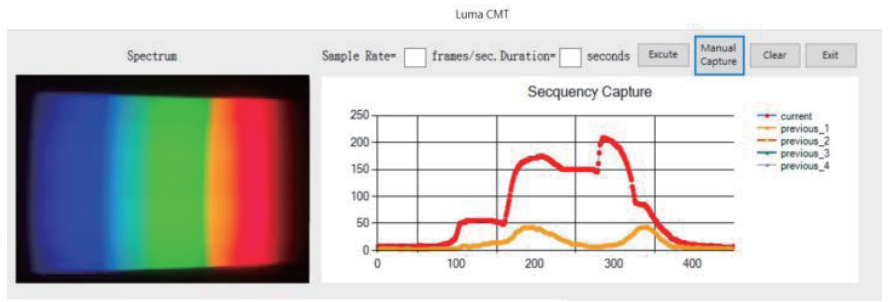

Fig. 2. (Color online) GUI of inspection system for online test. 
inspection system can measure the intensity of light passing through the CMT solution, which is compared with the time-elapsed intensity of the visible spectrum that was obtained using CCD. The sample rate can also be adjusted using the GUI during measurement. Since this instrument with no moving parts at all can measure the visible spectrum, it is very useful in cow-side tests on the farm.

Since the degree of gelation in the CMT is related to the SCC, optical and image processing technologies were used to measure the intensity of the light beam that passed through the SCC solution in a cuvette. Beer's Law yields the transmittance $(T)$ as

$$
T=I / I_{0} \text { or } \% T=100\left(I / I_{0}\right),
$$

where $I$ is the intensity of the light that passes through the SCC solution in a cuvette, and $\mathrm{I}_{0}$ is the intensity of the light that passes through the cuvette without a sample. The following equation yields the absorbance $(A)$.

$$
A=\log _{10}\left(I_{0} / I\right) \text { or } A=2-\log _{10}(\% T)
$$

The raw milk sample was hygienically collected from each Holstein cow and properly stored at $4{ }^{\circ} \mathrm{C}$ until analysis. A small volume of each sample $(50 \mathrm{ml})$ was extracted to determine its SCC by means of flow cytometry at the Testing Laboratory, Hsinchu Branch, Livestock Research Institute, Council of Agriculture, Taiwan. Raw milk $(6 \mathrm{ml})$ was combined 1:1 with an anionic surfactant (SDS: sodium dodecyl sulfate, $0.1 \mathrm{~g} / \mathrm{ml}$, in distilled water), and then $1 \mathrm{ml}$ of the CMT solution was extracted in a standard glass cuvette for optical inspection by obtaining its visible spectrum.

\section{Results and Discussion}

To calibrate the wavelengths of the visible spectrum of the halogen lamp passing through the cuvette without the CMT solution, green and red lasers, with wavelength bands around 543 and $633 \mathrm{~nm}$, respectively, were used. Figure 3 presents the wavelength of the halogen lamp, calibrated using green and red lasers.

To evaluate the CMT gelation score, which is related to SCC, the intensity of light that passed through the CMT solutions with various SCCs was measured, as presented in Fig. 4. The results included an intensity doublet around 517 and $609 \mathrm{~nm}$. Light at other wavelengths was significantly absorbed. The time required to make a single sample measurement was less than 2 min.

The intensity of the two wavelength bands around 517 and $609 \mathrm{~nm}$ in the visible spectrum was evaluated, and the effectiveness of each in measuring online of the CMT solution with various SCCs was evaluated, as presented in Fig. 5. The intensity of the light that passed through the CMT solution was found to be negatively correlated with the SCC. Wavelengths of around $517 \mathrm{~nm}$ were effective in SCC prediction, but those around $609 \mathrm{~nm}$ provided poor predictions at low SCC $\left(<10 \times 10^{4}\right.$ cells $\left./ \mathrm{ml}\right)$. 


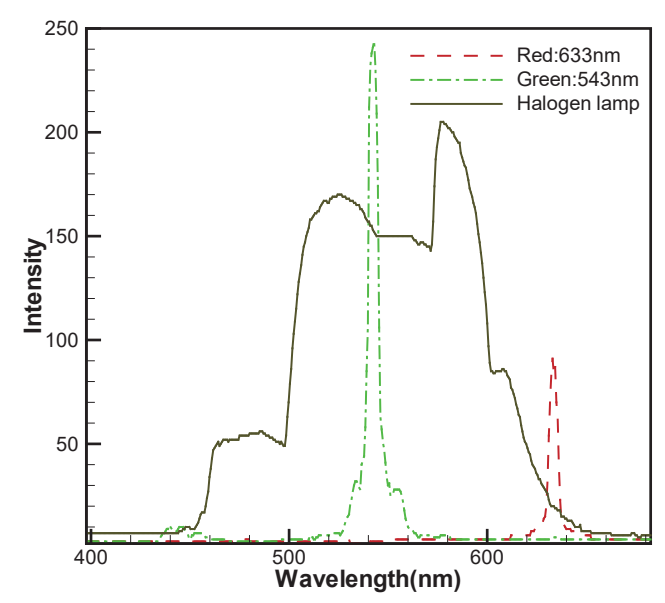

Fig. 3. (Color online) Spectrum of halogen lamp calibrated using green and red lasers.

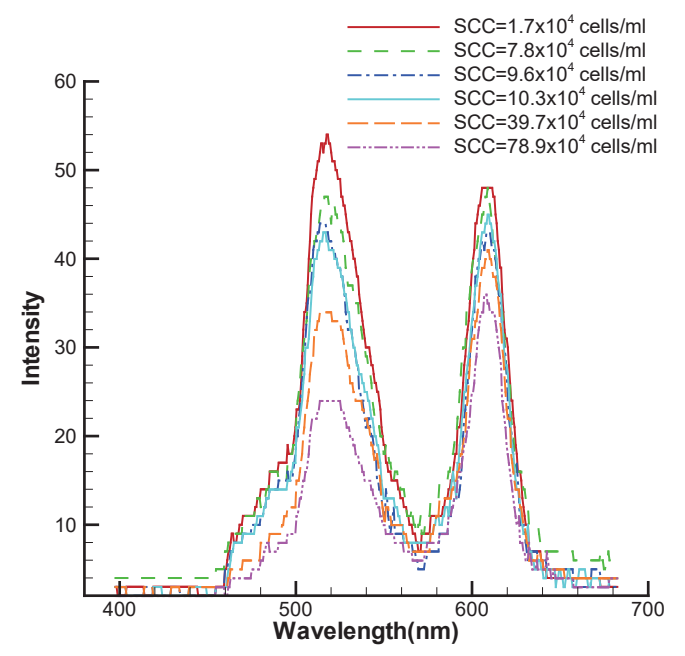

Fig. 4. (Color online) Intensity of visible spectrum of light through CMT solution with various SCCs.

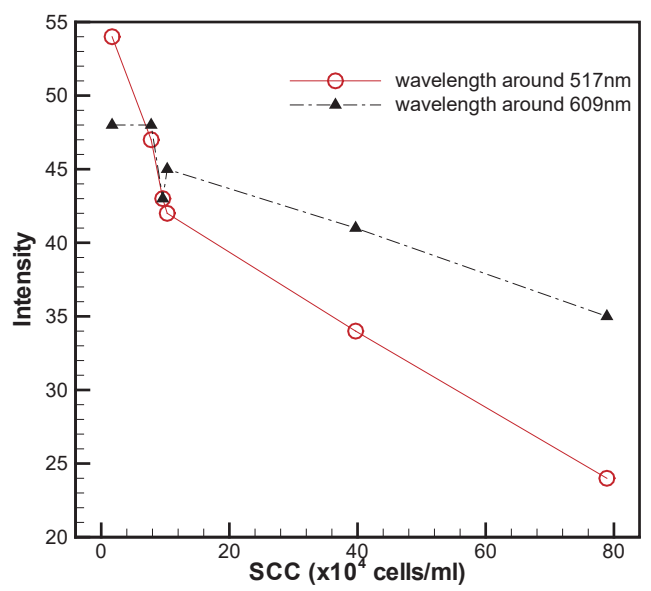

Fig. 5. (Color online) Relationship between intensity at different wavelengths and SCC.

Equations (1) and (2) yield the transmittance and absorption of light that passed through the CMT solution with different SCCs, as presented in Figs. 6 and 7. The relatively high transmittance and the relatively low absorption at low SCC were also expressed around 517 and $609 \mathrm{~nm}$.

The gelation of the CMT solution with a low SCC was measured, as shown in Fig. 8, indicating that the intensity of the light that passed through the CMT solution increased over time, mainly because the CMT reagent was SDS, which is known to be a strong protein denaturing detergent. This property allows SDS to sort proteins by size. SDS disassociates histones from DNA. ${ }^{(17)}$ Therefore, the CMT solution that is used with a low SCC does not significantly thicken at first, but becomes thinner over time, increasing the intensity of the light that passed through the CMT solution. 


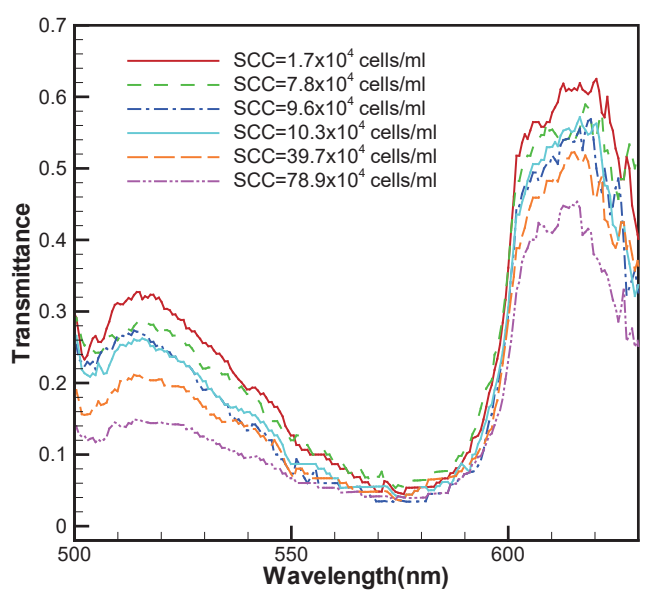

Fig. 6. (Color online) Transmittance spectrum of CMT solution with different SCCs.

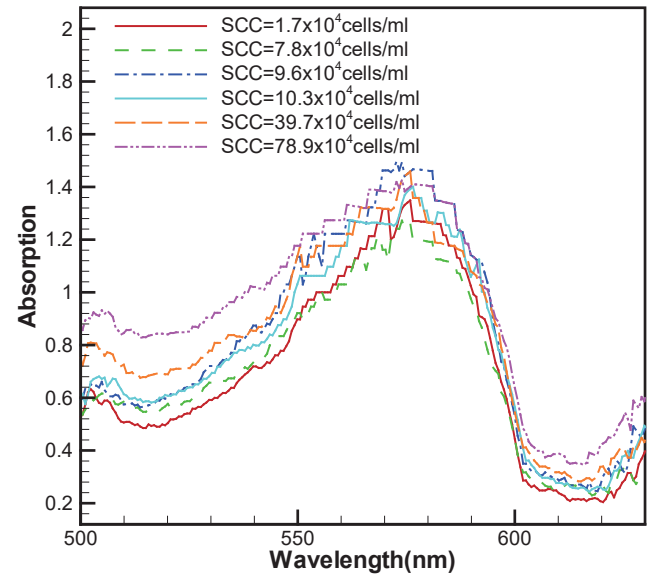

Fig. 7. (Color online) Absorption spectrum of CMT solution with different SCCs.

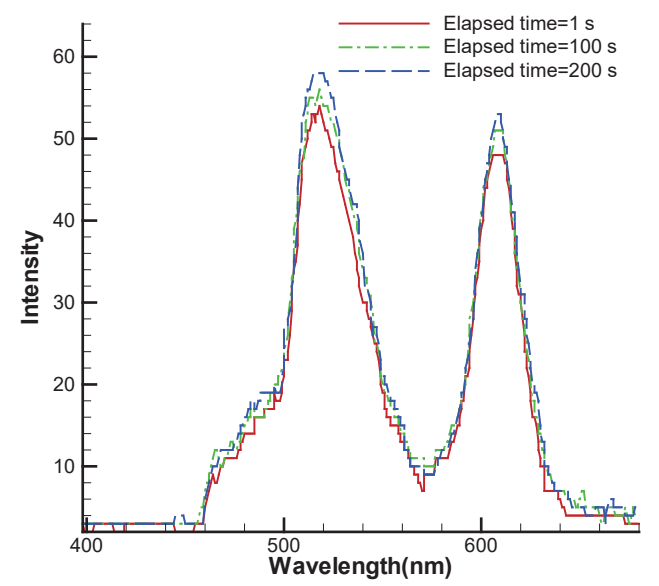

Fig. 8. (Color online) Intensity was increased with time at $S C C=1.7 \times 10^{4}(\mathrm{cell} / \mathrm{s} / \mathrm{ml})$.

On the basis of the above results, the CMT score can be objectively determined from the visible spectrum. The proposed inspection system may be an alternative method for diagnosing mastitis. In the future, sensitivity and specificity are further discussed in relation to the efficiency of the diagnosis of mastitis, and the experimental setup for detecting the score of SCC in milk will be made more compact, making it suitable for use in cow-side testing.

\section{Conclusions}

In conclusion, this work provided an optical system for objectively evaluating the score of a CMT solution, which is correlated with the SCC in milk, from a visible spectrum. Visible spectra revealed a negative correlation between the intensity of the light that passed through the CMT solution and the SCC in a CMT solution. The intensity of light with wavelengths of around 517 and $609 \mathrm{~nm}$, which passed through a CMT solution, was significantly correlated 
with SCC, providing an effective means of predicting the SCC using light with a wavelength of around $517 \mathrm{~nm}$, but the predictions of low SCCs $\left(<10 \times 10^{4}\right.$ cells $\left./ \mathrm{ml}\right)$ using a wavelength of around $609 \mathrm{~nm}$ were poor. The intensity of light that passed through a CMT solution was decreased systematically as the SCC increased. The intensity of this light increased over the duration of the gelation reaction, as the CMT solution became thinner. The proposed detection device setup will be made more compact for use in cow-side testing, and future work will discuss the sensitivity, specificity, and efficiency of the diagnosis of mastitis.

\section{Acknowledgments}

The authors would like to thank the Ministry of Science and Technology of Taiwan for financially supporting this research under Contract No. MOST 105-2221-E-276-001.

\section{References}

B. Heringstad, G. Klemetsdal, and J. Ruane: Livest. Prod. Sci. 64 (2000) 95.

C. Viguier, S. Arora, N. Gilmartin, K. Welbeck, and R. O’Kennedy: Trends Biotech. 27 (2009) 486.

M. J. Paape, W. P. Wergin, A. J. Guidry, and R. E. Pearson: J. Dairy Sci. 62 (1979) 135.

4 O. W. Schalm and D. Noorlander: J. Am. Vet. Med. Assoc. 130 (1957) 199.

5 N. C. Friggens, C. Ridder, and P. Løvendahl: J. Dairy Sci. 90 (2007) 5453.

6 G. Nageswarao and H. E. Calbert: J. Milk Food Technol. 32 (1969) 365.

7 M. G. Nichols and D. S. M. Phillips: Aust. J. Dairy Technol. 27 (1972) 134.

8 F. Kiermeier and K. Keis: Milchwissenschaft 19 (1964) 65.

9 W. Whittlestone, L. R. Fell, and H. De Langen: J. Milk Food Technol. 33 (1970) 351.

10 D. I. Thompson and D. S. Postle: J. Milk Food Technol. 27 (1964) 271.

11 F. H. J. Jaartsveld: Neth. Milk Dairy J. 16 (1962) 260.

12 V. Bottazzi: Il Latte 37 (1963) 755.

13 C. J. Verbeek, S. S. Xia, and D. Whyte: J. Dairy Res. 75 (2008) 385.

14 M. C. M. P. Brandão, A. P. Carmo, M. J. V. Bell, and V. C. Anjos: Rev. Inst. Latic. "Cândido Tostes", Mar/Abr, $\mathrm{n}^{\circ} 37365$ (2010) 30.

15 B. Aernouts, E. Polshin, J. Lammertyn, and W. Saeys: J. Dairy Sci. 94 (2011) 5315.

16 Z. Abdel-Salam, S. A. Attala, E. Daoud, and M. A. Harith: Dairy Sci. Technol. 95 (2015) 331.

17 D. Whyte, M. Walmsley, A. Liew, R. Claycomb, and G. Mein: J. Dairy Res. 72 (2005) 115.

\section{About the Authors}

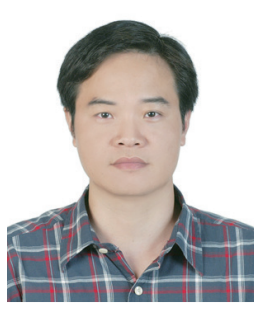

Chien-Hsing Chen received his M.E. and Ph.D. degrees in Mechanical Engineering from the National Cheng-Chung University, Taiwan, in 1997 and 2006, respectively. Since then, he has been with Information Technology Department of Meiho University, Taiwan, where he is currently an assistant professor. His research interests are in robotics, IoT, and sensors.

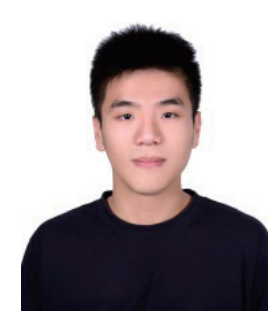

Chia-Heng Liou is a college student of the Department of Information Technology at Meiho University, Taiwan. 